\title{
Visualizing Local Electrical Properties of Composite Electrodes in Sulfide All-Solid-State Batteries by Scanning Probe Microscopy
}

\section{Misae Otoyama}

National Institute of Advanced Industrial Science and Technology (AIST)

\section{Takehiro Yamaoka}

Hitachi High-Tech Science Corporation

Hiroyuki Ito

Hitachi High-Tech Corporation

\section{Yuki Inagi}

Hitachi High-Tech Corporation

Atsushi Sakuda ( $\nabla$ saku@chem.osakafu-u.ac.jp )

Osaka Prefecture University https://orcid.org/0000-0002-9214-0347

\section{Masahiro Tatsumisago}

Osaka Prefecture University

\section{Akitoshi Hayashi}

Osaka Prefecture University https://orcid.org/0000-0001-9503-5561

\section{Article}

Keywords: Composite electrodes, scanning probe microscopy, SPM, batteries

Posted Date: August 24th, 2020

DOl: https://doi.org/10.21203/rs.3.rs-57856/v1

License: (9) This work is licensed under a Creative Commons Attribution 4.0 International License. Read Full License 


\section{Abstract}

Studies on local conduction paths in composite electrodes are essential to the realization of highperformance sulfide all-solid-state lithium batteries. Here, we directly evaluate the electrical properties of individual $\mathrm{LiNi}_{1 / 3} \mathrm{Mn}_{1 / 3} \mathrm{Co}_{1 / 3} \mathrm{O}_{2}$ (NMC) electrode active material particles in composite positive electrodes by scanning probe microscopy (SPM) techniques. Kelvin probe force microscopy (KPFM) and scanning spreading resistance microscopy (SSRM) were combined. The results indicated that all NMC particles exhibit a charged state with increasing potential, but low electronic conduction paths exist at point contacts of some NMC particles. Furthermore, the I-V characteristics measured by conductive-atomic force microscopy (C-AFM) suggest that these specific NMC particles show low charge-discharge reactivity. The results of the SPM techniques indicate that poor conduction locally limits the chargedischarge reactivity of electrode active materials, leading to the degradation of battery performance. Such SPM combination accelerates the morphological optimization of composite electrodes by facilitating the investigation of the intrinsic electrical properties of the electrodes.

\section{Introduction}

Studies on local conduction paths in composite electrodes are essential to the realization of highperformance sulfide all-solid-state lithium batteries. Here, we directly evaluate the electrical properties of individual $\mathrm{LiNi}_{1 / 3} \mathrm{Mn}_{1 / 3} \mathrm{Co}_{1 / 3} \mathrm{O}_{2}(\mathrm{NMC})$ electrode active material particles in composite positive electrodes by scanning probe microscopy (SPM) techniques. Kelvin probe force microscopy (KPFM) and scanning spreading resistance microscopy (SSRM) were combined. The results indicated that all NMC particles exhibit a charged state with increasing potential, but low electronic conduction paths exist at point contacts of some NMC particles. Furthermore, the $I-V$ characteristics measured by conductive-atomic force microscopy (C-AFM) suggest that these specific NMC particles show low charge-discharge reactivity. The results of the SPM techniques indicate that poor conduction locally limits the chargedischarge reactivity of electrode active materials, leading to the degradation of battery performance. Such SPM combination accelerates the morphological optimization of composite electrodes by facilitating the investigation of the intrinsic electrical properties of the electrodes.

All-solid-state lithium batteries (ASSLBs) are promising power sources for next-generation low-carbon societies $^{1,2}$. One of their primary advantages is their high safety factor as they replace flammable organic liquid electrolytes with non-flammable inorganic solid electrolytes (SEs). Recently, Kato et al. developed sulfide super-ionic conductors with a higher $\mathrm{Li}^{+}$ionic conductivity $\left(25 \mathrm{mS} \mathrm{cm}^{-1}\right)$ than conventional organic liquid electrolytes ${ }^{3}$. Bulk-type ASSLBs contain composite electrodes consisting of electrode active materials and SEs to form electron- and $\mathrm{Li}^{+}$-ion conduction paths. However, compared to conventional liquid electrolytes, it is difficult to generate a large number of conduction paths in ASSLBs because solidsolid interfaces tend to cause contact loss, resulting in inadequate electrochemical reactions.

To investigate the ionic and electronic conductivity of composite electrodes, Siroma et al. designed an AC impedance technique based on the transmission-line model ${ }^{4}$. Our group prepared ion-blocking and 
electron-blocking cells and used both $\mathrm{AC}$ and $\mathrm{DC}$ techniques for composite positive electrodes consisting of $\mathrm{LiNi}_{1 / 3} \mathrm{Mn}_{1 / 3} \mathrm{Co}_{1 / 3} \mathrm{O}_{2}(\mathrm{NMC})$ positive electrode active materials and $\mathrm{Li}_{3} \mathrm{PS}_{4}$ glass $\mathrm{SEs}$ of various compositions at $0 \%$ and $50 \%$ state-of-charge $(\mathrm{SOC})^{5}$. At SOC $0 \%$, the observed ionic conductivity was higher than the electronic conductivity. At SOC 50\%, electronic conductivity increased by more than one order of magnitude compared to that at SOC $0 \%$ and was higher than or equal to the ionic conductivity of the sample.

To analyze conduction networks in electrodes in detail, it is important to study local electrical properties in composite electrodes exhibiting complex morphologies. Investigating the reaction distribution in electrodes enables us to estimate local electrical properties indirectly. Recently, the SOC and Li distributions in composite positive electrodes in sulfide ASSLBs were studied by X-ray absorption spectroscopy ${ }^{6}$ and particle-induced $X$-ray emission/particle-induced gamma-ray emission ${ }^{7,8}$, respectively. We also demonstrated the reaction uniformity of composite electrodes by Raman imaging ${ }^{9-12}$. It could be inferred that charge-discharge reactions are not adequate in aggregated electrode active materials due to their small number of $\mathrm{Li}^{+}$-ion conduction paths.

Although scanning probe microscopy (SPM) can be used to directly investigate the electrical properties of composite electrodes $^{13-17}$, it has not been applied to study bulk-type sulfide ASSLBs because of challenges associated with handling sulfide SEs in air. Masuda et al. conducted operando Kelvin probe force microscopy (KPFM) on bulk-type oxide ASSLBs ${ }^{18,19}$. They investigated the internal electrical potential distribution in composite positive electrodes and proved that the conduction paths in these electrodes were disconnected in the first charging process.

Scanning spreading resistance microscopy (SSRM) and conductive-atomic force microscopy (C-AFM), which measure current and resistance distributions, respectively, are also based on SPM. These techniques have been used for investigating high-resistance areas to understand the degradation in electrode active materials ${ }^{15,20}$. Zhu et al. ${ }^{21}$ and Yang et al..$^{22}$ used a C-AFM technique of measuring I-V characteristics to investigate Li-ion diffusion energy barriers related to charge-discharge reactions in electrode active materials. They reported that the grain boundaries exhibited a lower barrier than did grain interiors in thin-film positive electrodes. To investigate the local electrical properties of composite electrodes in detail, SPM techniques such as KPFM, SSRM, and C-AFM should be employed simultaneously.

Herein, we showed for the first time monitoring of local electrical properties of sulfide composite positive electrodes in bulk-type ASSLBs by using various SPM techniques in a high vacuum state $\left(\sim 10^{-5} \mathrm{~Pa}\right)$. The same areas in the electrodes composed of both $\mathrm{NMC}$ and $\mathrm{Li}_{3} \mathrm{PS}_{4}$ SEs were evaluated by KPFM, SSRM, CAFM, and scanning electron microscopy-energy dispersive X-ray spectroscopy (SEM-EDX). We directly investigated the potential distribution in the electrodes with and without initial charging by KPFM to discuss about SOC distributions in each NMC particle. The distribution of local resistance in the composite electrodes was examined by SSRM. We evaluated changes in the local conductivity before 
and after charging from the results of resistance changes and compared them with previously reported findings on the variation in electronic conductivity ${ }^{5}$. Current distribution maps and $I-V$ curves of different NMC particles and the SE were obtained via C-AFM. We examined the reactivity of the NMC particles with higher and lower resistances in terms of their $I-V$ characteristics. Furthermore, voltage and resistance changes in the NMC particles were evaluated using KPFM and SSRM before and after the charge test. We also discussed the correlation between these changes and the morphology of the composite electrodes. The combination of these different SPM techniques for composite electrodes allowed us to successfully detect NMC particles that exhibited electrical properties different from those of others; these different characteristics are one of the main reasons for the poor conductivity observed in all-solid-state batteries.

\section{Results}

SPM analysis for composite positive electrodes. SPM measurements were conducted on composite positive electrodes consisting of $\mathrm{NMC}$ and $\mathrm{Li}_{3} \mathrm{PS}_{4}$ glass $\mathrm{SEs}$ before and after the initial charge test. Figure S1 (Supplementary information) shows the initial charge curve of the all-solid-state cell, which was charged to $4.4 \mathrm{~V}$ (vs. $\mathrm{Li}^{+} / \mathrm{Li}$ ) at $25^{\circ} \mathrm{C}$ at a current density of $0.13 \mathrm{~mA} \mathrm{~cm}^{-2}$; it exhibited an initial charge capacity of $164 \mathrm{mAh} \mathrm{g}^{-1}$. Before SPM measurements, we conducted ion milling on the cells to prepare flat samples. Subsequently, KPFM, C-AFM, SSRM, and SEM-EDX were conducted sequentially on the composite positive electrodes (Figure 1). An air-protected sample holder was used to transfer samples between the ion milling apparatus, SPM, and SEM. The negative electrode side was placed in contact with an insulating tape on the sample holder (details of the measurement conditions are included in the Methods section). Figure S2 shows an SEM image of the composite positive electrode after ion milling. Then SPM and SEM-EDX measurements were conducted for the same areas on the electrodes near the SE layer. The potential, current, and resistance distributions were measured by KPFM, C-AFM, and SSRM, respectively, in the composite positive electrodes. In the setup used for both C-AFM and SSRM, a bias voltage of $-2 \mathrm{~V}$ was applied on the sample holder. In general, the SSRM technique results in a wide measurement range of 7 orders of magnitude, thereby enabling the analysis of composite materials exhibiting large differences in resistance. Meanwhile, by using sweeping bias voltage, C-AFM yields the I$V$ characteristics of the composite electrodes and SEs. We investigated $I-V$ characteristics of the SE separator layer by C-AFM and confirmed that the bias voltage of $-2 \mathrm{~V}$ did not induce SE decomposition (Figure S3; see details in the Methods section). Finally, a bias voltage of $-2 \mathrm{~V}$ was found to be suitable for detecting small currents $(10 \mathrm{pA})$ and high resistances $\left(10^{9} \Omega\right)$ in composite electrodes.

Figures 2 and 3 show the SEM image, EDX mappings, and SSRM, C-AFM, and KPFM images of the composite electrode before cell operation and after the initial charge test, respectively. $\mathrm{S}$ and $\mathrm{Ni}$ could be detected in the EDX maps of SE and NMC, respectively. Furthermore, all SSRM, C-AFM, and KPFM images overlapped both the NMC and SE areas, indicating that all measurements could be carried out successfully in the same area. The SSRM, C-AFM, and KPFM images of the electrode captured before the charge test indicated minimal differences between individual NMC particles (Figure 2(d)-(f)). In the SSRM image, it can be observed that the resistance at the center of the NMC particles and SE/NMC 
interfaces was lower than that in other NMC areas. However, the range of these values was $1.8-2.2 \times 10^{9}$ $\Omega$, indicating that all areas in the composite electrodes showed a similar resistance before charging. In contrast, the resistance of some delithiated NMC particles varied from that of other NMC particles in the charged sample (Figure 3(d)). Most of the NMC particles contacted each other sufficiently and exhibited a resistance of $10^{7} \Omega$, while some NMC particles 'point-contacted' with other particles as shown in the SEM image (Figure 3(a)) with a yellow broken line; these particles exhibited a higher resistance of $10^{9} \Omega$. Such higher resistance may be attributed to poor electronic conduction due to inadequate contact among these NMC particles. In the C-AFM image (Figure 3(e)), the contrast of these NMC particles and SE was similar, indicating that NMC particles in minimal contact with other particles exhibited lower electronic conductivity due to their higher resistance. Meanwhile, the resistance of SE remained constant at $10^{9} \Omega$ after charging.

The contact potential difference $\left(\mathrm{V}_{\mathrm{CPD}}\right)$ between the tip and positive electrode was measured by KPFM. As shown in Figure 1, the negative electrode side was opened and the positive electrode was in electrical contact with the sample holder. To compare KPFM images before and after the charge test, $\mathrm{V}_{\mathrm{CPD}}$ was converted into $\mathrm{V}_{\mathrm{CPD}}$ ' by adding the open circuit voltage (OCV), as described in the Methods section. Hereafter, we shall discuss KPFM results in terms of $\mathrm{V}_{\mathrm{CPD}}$ '. Figures $2(\mathrm{f})$ and $3(\mathrm{f})$ depict the observed $\mathrm{V}_{\mathrm{CPD}}{ }^{\prime}$ values. In the composite electrodes before charging, the $\mathrm{V}_{\mathrm{CPD}}$ ' values of NMC and SE were 2.06-2.12 and $2.22 \mathrm{~V}$, respectively; these values increased to $3.11-3.29$ and $2.76 \mathrm{~V}$, respectively, after charging. When compared to $\mathrm{SE}, \mathrm{NMC}$ exhibited a larger difference in $\mathrm{V}_{\mathrm{CPD}}{ }^{\prime}(1.1 \mathrm{~V})$ before and after charging. Although $\mathrm{V}_{\mathrm{CPD}}$ ' does not correspond with cell voltage quantitatively, we considered that KPFM measurements can be used to evaluate potential changes qualitatively as the $\mathrm{V}_{\mathrm{CPD}}$ ' of $\mathrm{NMC}$ increased after charging in response to an increase in the cell voltage. In the present study, we discuss the potential distribution in each electrode before and after the charge test.

I-V characteristics of electrodes measured by C-AFM. The I- $\mathrm{V}$ characteristics of NMC particles and the SEs were compared using C-AFM (Figure 4). Figure 4(b) shows the I-V curves of NMC ((a1) and (a2)) and SE ((a3) and (a4)) from the C-AFM image before charging (Figure 4(a)). The $l-V$ curves of active electrode materials yield information about their electrical properties at the measuring point ${ }^{21,22}$. Although this experimental technique is typically applied to thin-film electrodes, we assumed that it could be applied to bulk-type ASSLBs in order to investigate the local charge-discharge properties of a single electrode active material particle in the presence of SE. While the current in the SE was $0 \mathrm{~A}$, the NMC particles displayed different $I-V$ curves before charging owing to their different charge-discharge reactivities at each single point; NMC (a1) responded with a higher current than (a2). As shown in Figure 4(b), I-V characteristics are locally different even before charging. In the composite electrodes after charging, NMC particles exhibiting higher resistance ((c1) and (c2)) show completely different $I-V$ curves compared to those exhibiting lower resistance ((c3) and (c4)). The latter show higher current responses due to their lower resistances, suggesting that charge-discharge reactions occurred easily at these NMC particles. 
Investigation of the $I-V$ characteristics of electrodes by C-AFM can help us understand the local reactivities in charge-discharge reactions.

\section{Potential and resistance distributions of individual NMC particles measured by KPFM and SSRM. We} evaluated the electrical properties of individual NMC particles. We selected 14 NMC particles from the KPFM and SSRM images as shown in Figures 5(a)-(d) and evaluated the average $\mathrm{V}_{\mathrm{CPD}}$ ' and resistance of each particle; the obtained results are shown in Figure $5(\mathrm{e})$. Before charging, the $\mathrm{V}_{\mathrm{CPD}}$ ' and resistance values of all the NMC particles were $\sim 2.1 \mathrm{~V}$ and $2.1 \times 10^{9} \Omega$, respectively. After charging, the value of $\mathrm{V}_{\mathrm{CPD}}$ ' increased to 3.1-3.3 V, suggesting the occurrence of delithiation in all NMC. The resistance of most of the NMC particles decreased to $\sim 10^{7}-10^{8} \Omega$, indicating an increase in their electronic conductivity. This behavior corresponded with our previous observations that the electronic conductivity of composite positive electrodes increased by 1-2 orders of magnitude after charging ${ }^{5}$. However, the resistances of three NMC particles, numbered 03,08 , and 10 , were $10^{9} \Omega$ even after charging. As described earlier, these particles had minimal contact with other NMC particles. It can be inferred from the $\mathrm{V}_{\mathrm{CPD}}$ ' results that delithiation occurred in each $\mathrm{NMC}$ particle in the charged electrode because $\mathrm{V}_{\mathrm{CPD}}$ ' of observed $\mathrm{NMC}$ particles increased after charging. However, the local electronic conductivity was not uniform as some NMC particles showed higher resistance after charging. This phenomenon was prominent in the case of NMC particles in 'point' contact with other particles without any conductive additive. At high current densities, it is likely that inhomogeneous electronic conduction degrades battery performance due to current concentration. Increasing the amount of electrode active materials ${ }^{5}$ and tailoring their sizes ${ }^{23,24}$ can improve the electronic conductivity of carbon-free composite electrodes. However, electrode utilization is limited in the case of no or insufficient quantity of conductive additives. Moreover, some reports state that all-solid-state cells degrade in the presence of carbon ${ }^{25}$, which necessitates the optimization of these composite electrodes. Information on local electronic conduction provided by SSRM makes it a powerful tool to optimize electrode design. Our previous studies using Raman imaging indicated that aggregated electrode active materials show a low SOC as they contain only a small number of ionic conduction paths ${ }^{9}$. In contrast, in the present study, SSRM showed that minimal contact between NMC particles resulted in a low local electronic conductivity.

Subsequently, we analyzed the resistance and $\mathrm{V}_{\mathrm{CPD}}$ ' distribution in the cross-sectional direction. The NMC particles are numbered from the current collector (CC) side toward the SE layer as shown in Figures 5(a)(d). Figure 5(f) shows the variation in the resistance of the NMC particles before and after the charge test. As described earlier, all the NMC particles in the measurement zone exhibited a similar resistance before charging. At the end of the charge test, the resistance of most of the NMC particles decreased except for the particles numbered 03,08 , and 10 , which did not have much contact with other NMC particles. Figure $5(f)$ indicates that there was no resistance gradient in the cross-sectional direction. However, the $\mathrm{V}_{\mathrm{CPD}}{ }^{\prime}$ distribution was different from that corresponding to resistance of NMC particles. The $\mathrm{V}_{\mathrm{CPD}}$ ' values of all the NMC particles before and after the charge test are plotted in Figures S4(a) and (b), respectively. Before charging, there was no gradient in the $\mathrm{V}_{\mathrm{CPD}}$; in contrast, after charging, it increased gradually from 
the SE layer side to the CC side except for particles 03 and 08 , which exhibited a higher resistance. The difference in $\mathrm{V}_{\mathrm{CPD}}$ ' values on the SE layer and CC sides was $\sim 0.2 \mathrm{~V}$ (except for particles 03 and 08), indicating a potential gradient in the cross-sectional direction after the charge test. In our previous studies using Raman imaging and optical microscopy, we observed inhomogeneous SOC distributions in composite electrodes after charging 9,10,12,26,27. The results showed that charge-discharge reactions proceeded preferentially from the SE-layer side because the rate-determining step was related to $\mathrm{Li}^{+}$-ion conduction, indicating that the potential of NMC increased from the SE-layer side. In contrast, the results in Figure S4(b) indicate that NMC particles on the SE-layer side exhibit a lower potential than those on the CC side. In this study, the measurement area was focused on $15 \mu \mathrm{m}^{2}$ near the SE layer of the $50 \mu \mathrm{m}$ thick electrode layer (Figure S2). Therefore, KPFM analysis indicates a local potential gradient. To further investigate the presence of a potential gradient across the entire composite electrode, in situ and widerange measurements are required. Figure S4(b) indicates that a potential gradient remained after the charge test in the all-solid-state cells. Moreover, Tanida et al. reported that after relaxation in charged cells with an organic liquid electrolyte, the $\mathrm{SOC}$ of $\mathrm{LiCoO}_{2}$ electrodes became uniform with a local potential difference as the driving force ${ }^{28}$. The behavior of all-solid-state cells is different from that of conventional batteries with an organic liquid electrolyte, possibly because the former contains fewer conductive networks ${ }^{19,23}$. From Figures 5 and S4, it could be inferred that the inhomogeneous resistance and potential distributions are likely to be dependent on the percolation of NMC particles and their distance from the SE layer, respectively.

\section{Discussion}

To investigate the electrical properties of individual NMC particles in composite positive electrodes, we conducted SPM and SEM-EDX analyses on the same areas in the electrodes of sulfide bulk-type ASSLBs before and after the charge test. The KPFM indicated that the potentials of all NMC particles in the measurement area increased after the charge test, suggesting the delithiation of NMC. The resistance distribution measured by SSRM showed that the resistances of almost all the NMC particles decreased by more than one order of magnitude. This result corresponded with the previous report indicating that the ionic conductivity of composite electrodes increased after charging. However, some NMC particles, which had little contact with other NMC particles, showed high resistance after charging. Therefore, the combination of KPFM and SSRM revealed that charge reactions occurred in the composite electrode, but low electronic conduction paths were formed locally. These specific low electronic conduction paths are likely to induce battery degradation at high current density. Recent papers have discussed particle sizes of electrode active materials and the necessity of conductive additives in terms of electronic conduction paths in composite electrodes. Investigations of local electronic conduction by SSRM and potential distributions by KPFM contribute to facilitate such optimization of composite electrodes.

Furthermore, for the first time, we employed C-AFM on composite electrodes in bulk-type ASSLBs to investigate their $I-V$ characteristics. The SE showed a small current response at high bias voltages, suggesting low electronic conductivity and oxidative decomposition. The reactivity of charge-discharge 
reactions of $\mathrm{NMC}$ was investigated by comparing the $I-V$ characteristics of individual NMC particles. In the NMC particles for which SSRM measured lower resistance, higher current responses were recorded, suggesting their high preference for charge-discharge reactions. Therefore, the combination of C-AFM and SSRM demonstrated that decreasing the amount of NMC particles exhibiting high resistance significantly increased the charge-discharge reactivity of the electrode.

Finally, combining different SPM techniques enables us to investigate the electrical properties of electrode active materials in composite electrodes, such as their potential, current, and resistance distributions, and $I-V$ characteristics, directly and to diagnose poor conduction paths. This may help in formulating guidelines to improve electronic conduction in composite electrodes for bulk-type ASSLBs. For further understanding of resistance and potential distribution formations during charge-discharge reactions, in situ measurements will be our future research. Moreover, combining other measurements such as Raman imaging will help us to gain a direct relationship between SOC and electrical properties of electrode active materials.

\section{Methods}

Fabrication of all-solid-state lithium cells. $75 \mathrm{Li}_{2} \mathrm{~S} \cdot 25 \mathrm{P}_{2} \mathrm{~S}_{5}$ (mol\%) glass SEs were prepared by mechanical milling of $\mathrm{Li}_{2} \mathrm{~S}$ (Mitsuwa Chemicals Co., Ltd, 99.9\%) and $\mathrm{P}_{2} \mathrm{~S}_{5}$ (Aldrich, 99\%) in a dry Ar atmosphere as described in a previous study ${ }^{29}$. The composite positive electrodes were prepared by grinding $\mathrm{LiNbO}_{3}$ coated NMC (Toda Kogyo Corp.) and SE powder together using a mortar. The weight and volume ratios of NMC and SE were 75:25 (wt.\%) and 54:46 (vol.\%), respectively. All-solid-state cells (10 mm diameter) were constructed by uniaxial pressing. Initially, SE powder $(50 \mathrm{mg})$ was pelletized, after which the NMC composite positive electrode powder $(11 \mathrm{mg})$ was ground on the SE pellet and pressed slightly. Stainless steel powder (SUS304L, Kojundo Chemical Laboratory Co., Ltd.) $(100 \mathrm{mg})$ was placed on the two-layer pellet and pressed at $360 \mathrm{MPa}$ for $5 \mathrm{~min}$. Indium foil (99.999\%, thickness of $0.1 \mathrm{~mm}$; Furuuchi Chemical Corp.) ( $\phi=9 \mathrm{~mm}$ ), lithium foil ( $99.9 \%$, thickness of $0.25 \mathrm{~mm}$; Furuuchi Chemical Corp.) (1 mg), and stainless steel foil (SUS316L; Nilaco Corp.) ( $\phi=10 \mathrm{~mm}$ ) were attached to the SE separator side of the three-layer pellet and pressed at $180 \mathrm{MPa}$. The pellet was then sealed in an Al laminate pouch cell with $\mathrm{Al}$ and $\mathrm{Ni}$ tubs in an Ar atmosphere. The all-solid-state cell was charged to $4.4 \mathrm{~V}\left(\mathrm{vs}\right.$. $\left.\mathrm{Li}^{+} / \mathrm{Li}\right)$ at a current density of $1.3 \mathrm{~mA} \mathrm{~cm}^{-2}$ at $25^{\circ} \mathrm{C}$ using a charge-discharge measuring device (BTS-2004; Nagano Co., Ltd.).

Ion milling of all-solid-state cells. The pristine and charged cells were cut with razors and set in an airprotected sample holder in an Ar atmosphere. To obtain flat cross-sections, ion milling (ArBlade5000; Hitachi High-Tech Corp.) was conducted on the cells with cooling at $-100^{\circ} \mathrm{C}$. We confirmed that the OCV of the cells did not change before and after ion milling.

KPFM analysis. After ion milling, KPFM was conducted on the NMC composite positive electrodes using a scanning probe microscope (AFM5300E; Hitachi High-Tech Corp.). All SPM measurements were carried out without dismounting the cells from the sample holder in a high vacuum $\left(\sim 10^{-5} \mathrm{~Pa}\right)$. For KPFM 
analysis, the negative electrode side was opened and the positive electrode was in electrical contact with the sample holder. This is because a wide flat sample can be obtained when ion milling is performed from the positive electrode side, which is in direct contact with the sample holder without an insulating tape. In the present setup (Figure 1), the positive electrode was connected to the ground and $\mathrm{V}_{\mathrm{OCV}}$ with a minus sign for the cells was expected on the negative electrode side. Generally, KPFM measures $V_{C P D}$ between the sample positive electrode and probe tip. In the case of cells displaying an electromotive force, the observed KPFM voltage included cell voltage as well. Note that measurement areas in the positive electrodes were near ground in the experimental setup, owing to which it was difficult to examine voltage changes before and after the charge test. To overcome this issue, $\mathrm{V}_{\mathrm{CPD}}$ was converted to $\mathrm{V}_{\mathrm{CPD}}$ ' (= $\mathrm{V}_{\mathrm{CPD}}+\mathrm{V}_{\mathrm{OCV}}$ ) by adding $\mathrm{V}_{\mathrm{OCV}}$ before and after the charge test (1.16 and $3.74 \mathrm{~V}$, respectively) to $\mathrm{V}_{\mathrm{CPD}}$, while assuming that the negative electrode was grounded and the positive electrode was opened. We used a Rh-coated Si cantilever (SI-DF3-R; Hitachi High-Tech Corp.) with a resonance frequency of $23-31 \mathrm{kHz}$ and spring constant of $\sim 1.5 \mathrm{~N} \mathrm{~m}^{-1}$; furthermore, an AC voltage of $0.5 \mathrm{~V}$ was applied at a frequency of 26.0 $\mathrm{kHz}$ between the probe and sample holder. We evaluated the $\mathrm{V}_{\mathrm{CPD}}$ ' of each NMC particle in the observation area by selecting each individual particle from KPFM images, generating the corresponding $\mathrm{V}_{\mathrm{CPD}}$ ' histogram, and evaluating $\mathrm{V}_{\mathrm{CPD}}$ ' at the peak of the histogram.

C-AFM, SSRM, and SEM-EDX analyses. After KPFM evaluation, C-AFM was carried out at a bias voltage of $-2 \mathrm{~V}$ on the same areas in the composite electrodes. The Sampling Intelligent Scan (SIS) ${ }^{30}$ mode was used for SSRM and C-AFM measurements. A boron-doped diamond-coated Si cantilever (SI-DF40-CD; Hitachi High-Tech Corp.) was used for these studies. The $I-V$ curves of individual NMC particles and SE in the composite electrodes and the SE layer were recorded in the bias voltage sweep range of -2 to $2 \mathrm{~V}$ at a scan rate of $4 \mathrm{~V} \mathrm{~s}^{-1}$. The $I-V$ characteristics of the SE separator layer were investigated by C-AFM (Figure S3) to select a suitable bias voltage. A bias voltage was applied at four points on the SE separator layer and swept from -2 to $2 \mathrm{~V}$; Figure S3(b) shows the resultant changes in current. In the range of -2 to $1 \mathrm{~V}$, there was almost zero current, after which it gradually increased to $1 \mathrm{pA}$ during the subsequent voltage sweep to $2 \mathrm{~V}$. These changes indicated that SE was decomposed by oxidation. We selected a bias voltage of $-2 \mathrm{~V}$ for C-AFM and SSRM measurements to prevent SE decomposition.

After C-AFM analysis, SSRM tests were conducted on the same areas with the same cantilever and measurement setup as those in the C-AFM scheme. We investigated the resistance of each NMC particle in the observation area in a manner similar to that used for evaluating the $\mathrm{V}_{\mathrm{CPD}}$ ' of NMC particles.

After SSRM testing, the morphology of the electrodes was observed by SEM-EDX (FE-SEM: Regulus8100, Hitachi High-Tech Corp.; EDX: Ultim Max $\left(100 \mathrm{~mm}^{2}\right)$, Oxford Instruments). In all processes (ion milling to SEM-EDX), the same air-protected sample holder with an 0-ring was used.

\section{Declarations}

\section{Acknowledgements}


This research was supported by JSPS KAKENHI (Grant Numbers 18H05255 and 17J09397).

\section{Author Contributions}

M. O. developed the idea and constructed the all-solid-state cells. T. Y., H. I., and Y. I. conducted ion milling and SEM-EDX and SPM measurements. A. S., M. T., and A. H. supervised the project. All authors discussed the results and prepared the manuscript.

Competing Interests: There are no competing interests to declare.

Materials \& Correspondence: Information relevant to this study may be requested from Dr. Atsushi Sakuda (saku@chem.osakafu-u.ac.jp).

\section{References}

1. Hayashi, A., Sakuda, A. \& Tatsumisago, M. Development of sulfide solid electrolytes and interface formation processes for bulk-type all-solid-state Li and Na batteries. Energy Res. 4, 25 (2016).

2. Janek, J. \& Zeier, W. G. A solid future for battery development. Nature Energy 1, 16141 (2016).

3. Kato, Y. et al. High-power all-solid-state batteries using sulfide superionic conductors. Nature Energy 1, 16030 (2016).

4. Siroma, Z. et al. AC impedance analysis of ionic and electronic conductivities in electrode mixture layers for an all-solid-state lithium-ion battery. Power Sources 316, 215-223 (2016).

5. Asano, T., Yubuchi, S., Sakuda, A., Hayashi, A. \& Tatsumisago, M. Electronic and ionic conductivities of $\mathrm{LiNi}_{1 / 3} \mathrm{Mn}_{1 / 3} \mathrm{Co}_{1 / 3} \mathrm{O}_{2}-\mathrm{Li}_{3} \mathrm{PS}_{4}$ positive composite electrodes for all-solid-state lithium batteries. Electrochem. Soc. 164, A3960-A3963 (2017).

6. Chen, K. et al. Morphological effect on reaction distribution influenced by binder materials in composite electrodes for sheet-type all-solid-state lithium-ion batteries with the sulfide-based solid electrolyte. Phys. Chem. C123, 3292-3298 (2019).

7. Yoshino, K. et al. Lithium distribution analysis in all-solid-state lithium battery using microbeam particle-induced X-ray emission and particle-induced gamma-ray emission techniques. J. PIXE 27, 1120 (2017).

8. Yamada, Y. et al. Ex-situ analysis of lithium distribution in a sulfide-based all-solid-state lithium battery by particle-induced X-ray and gamma-ray emission measurements. Electrochemistry $88,45-$ 49 (2020).

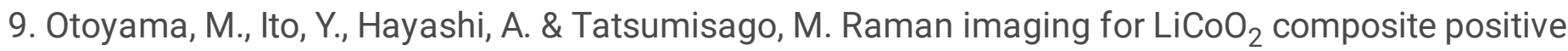
electrodes in all-solid-state lithium batteries using $\mathrm{Li}_{2} \mathrm{~S}-\mathrm{P}_{2} \mathrm{~S}_{5}$ solid electrolytes. Power Sources 302 , 419-425 (2016).

10. Otoyama, M., Ito, Y., Hayashi, A. \& Tatsumisago, M. Investigation of state-of-charge distributions for $\mathrm{LiCoO}_{2}$ composite positive electrodes in all-solid-state lithium batteries by Raman imaging. Lett. 45, 810-812 (2016). 
11. Otoyama, M., Ito, Y., Hayashi, A. \& Tatsumisago, M. Raman Spectroscopy for $\mathrm{LiNi}_{1 / 3} \mathrm{Mn}_{1 / 3} \mathrm{Co}_{1 / 3} \mathrm{O}_{2}$ composite positive electrodes in all-solid-state lithium batteries. Electrochemistry 84, 812-814 (2016).

12. Otoyama, M., Ito, Y., Sakuda, A., Tatsumisago, M. \& Hayashi, A. Reaction uniformity visualized by Raman imaging in the composite electrode layers of all-solid-state lithium batteries. Chem. Chem. Phys. 22, 13271 (2020).

13. Elazari, R. et al. Morphological and structural studies of composite sulfur electrodes upon cycling by HRTEM, AFM and Raman spectroscopy. Electrochem. Soc. 157, A1131-A1138 (2010).

14. Kim, S. H. et al. Nanoscale electrical degradation of silicon-carbon composite anode materials for lithium-ion batteries. ACS Appl. Mater. Interfaces 10, 24549-24553 (2018).

15. Park, S. Y. et al. Probing electrical degradation of cathode materials for lithium-ion batteries with nanoscale resolution. Nano Energy 49, 1-6 (2018).

16. Wang, S. et al. Advances in understanding materials for rechargeable lithium batteries by atomic force microscopy. Energy Environ. Mater. 1, 28-40 (2018).

17. Kempaiah, R., Vasudevamurthy, R. \& Subramanian, A. Scanning probe microscopy based characterization of battery materials, interfaces, and processes. Nano Energy 65, 103925 (2019).

18. Masuda, H., Ishida, N., Ogata, Y., Ito, D. \& Fujita, D. Internal potential mapping of charged solid-statelithium ion batteries using in situ Kelvin probe force microscopy. Nanoscale 9, 893-898 (2017).

19. Masuda, H., Matsushita, K., Ito, D., Fujita, D. \& Ishida, N. Dynamically visualizing battery reactions by operando Kelvin probe force microscopy. Chem. 2, 140 (2019).

20. Nagpure, S. C., Bhushan, B., Babu, S. \& Rizzoni, G. Scanning spreading resistance characterization of aged Li-ion batteries using atomic force microscopy. Scripta Materialia 60, 933-936 (2009).

21. Zhu, X. et al. Direct observation of lithium-ion transport under an electrical field in $\mathrm{Li}_{x} \mathrm{CoO}_{2} \mathrm{Sci}$. Rep. 3 , 1084 (2013).

22. Yang, S., Yan, B., Lu, L. \& Zeng, K. Grain boundary effects on Li-ion diffusion in a $\mathrm{Li}_{2} \mathrm{Co}_{0.13} \mathrm{Ni}_{0.13} \mathrm{Mn}_{0.54} \mathrm{O}_{2}$ thin film cathode studied by scanning probe microscopy techniques. RSC Adv. 6, 94000-94009 (2016).

23. Strauss, F. et al. Impact of cathode material particle size on the capacity of bulk-type all-solid-state batteries. ACS Energy Lett. 3, 992-996 (2018).

24. Shi, T. et al. High active material loading in all-solid-state battery electrode via particle size optimization. Energy Mater. 10, 1902881 (2019).

25. Zhang, W. et al. The detrimental effects of carbon additives in $\mathrm{Li}_{10} \mathrm{GeP}_{2} \mathrm{~S}_{12}$-based solid-state batteries. ACS Appl. Mater. Interfaces 9, 35888-35896 (2017).

26. Otoyama, M., Sakuda, A., Hayashi, A. \& Tatsumisago, M. Optical microscopic observation of graphite composite negative electrodes in all-solid-state lithium batteries. Solid State lonics 323, 123-129 (2018).

27. Otoyama, M., Sakuda, A., Tatsumisago, M. \& Hayashi, A. Operando confocal microscopy for dynamic changes of $\mathrm{Li}^{+}$ion conduction path in graphite electrode layers of all-solid-state batteries. Phys. 
Chem. Lett. 11, 900-904 (2020).

28. Tanida, $\mathrm{H}$. et al. Elucidating the driving force of relaxation of reaction distribution in $\mathrm{LiCoO}_{2}$ and $\mathrm{LiFePO}_{4}$ electrodes using X-ray absorption spectroscopy. Phys. Chem. C 120, 4739-4743 (2016).

29. Hayashi, A., Hama, S., Minami, T. \& Tatsumisago, M. Formation of superionic crystals from mechanically milled $\mathrm{Li}_{2} \mathrm{~S}-\mathrm{P}_{2} \mathrm{~S}_{5}$ Electrochem. Commun. 5, 111-114 (2003).

30. Yasutake, M., Watanabe, K., Wakiyama, S., \& Yamaoka, T. Critical dimension measurement using new scanning mode and aligned carbon nanotube scanning probe microscope tip. J. Appl. Phys. 45, 1970-1973 (2006).

\section{Figures}

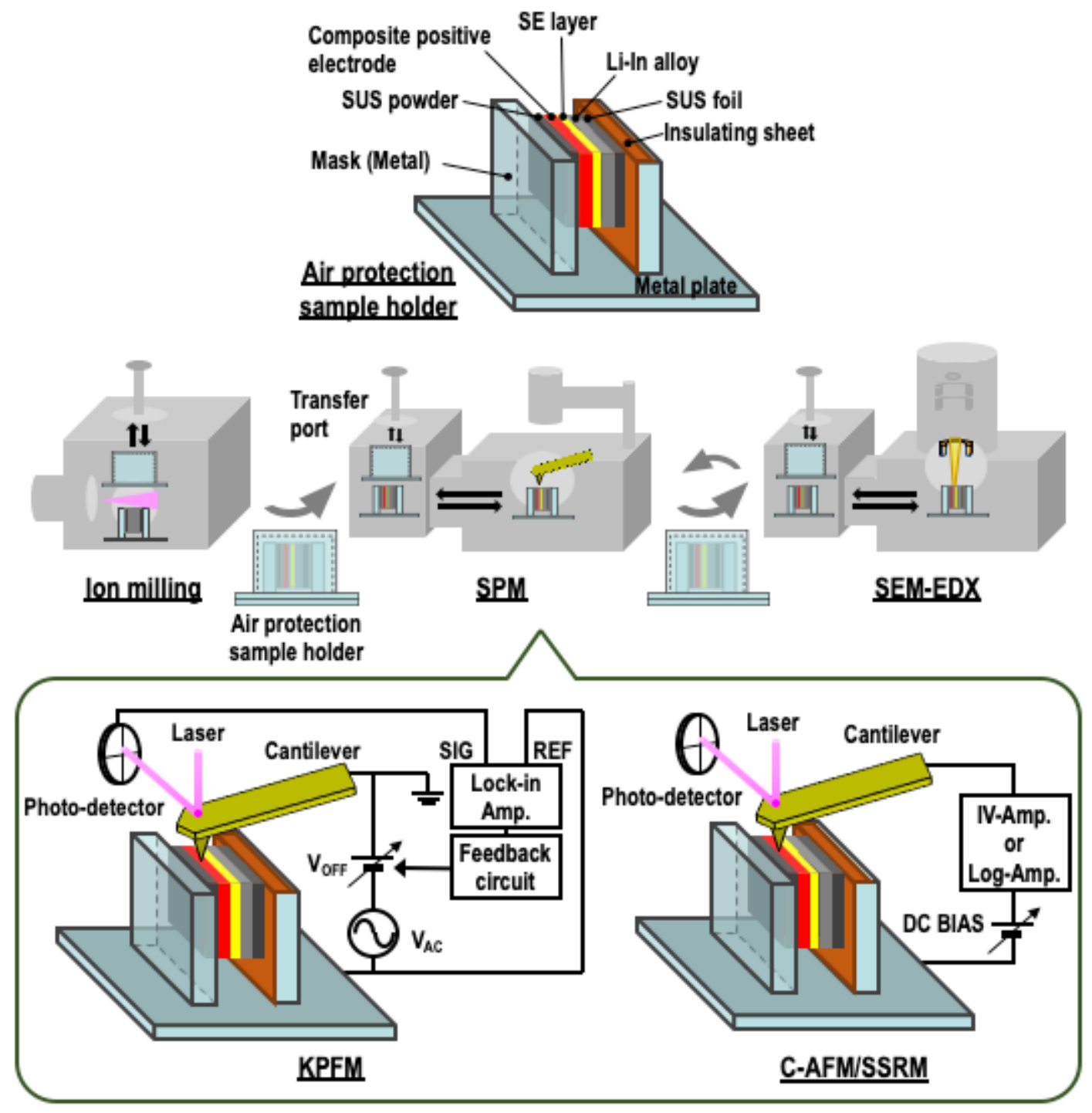

Figure 1 
Ion milling treatment and SPM analyses. Schematic illustration of the ion milling treatment and KPFM, CAFM, SSRM, and SEM-EDX analyses. Samples were transferred in an air-protected sample holder and the same areas were analyzed by KPFM, C-AFM, SSRM, and SEM-EDX.

(a) SEM

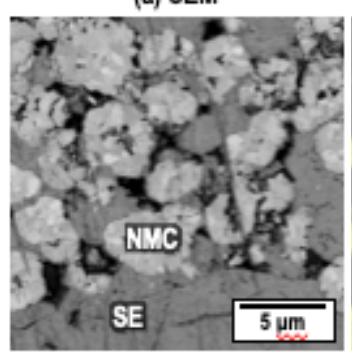

(d) SSRM

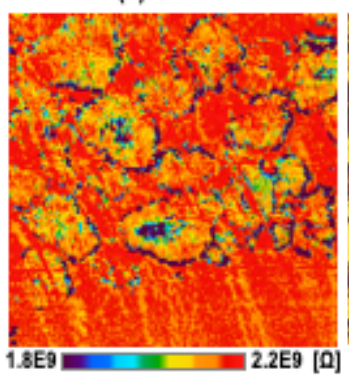

(b) EDX mapping

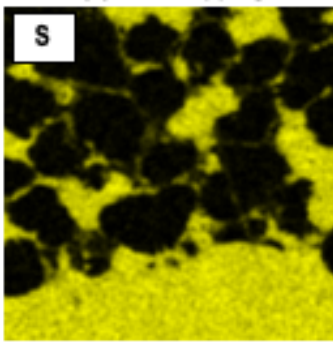

(e) C-AFM

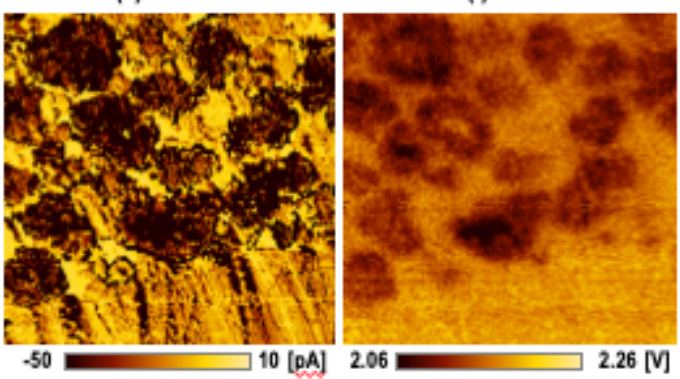

(c) EDX mapping

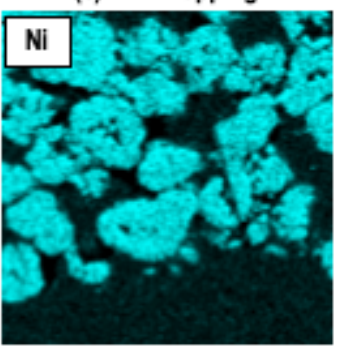

(f) KPFM

\section{Figure 2}

SPM analysis of the composite positive electrode before charging. (a) SEM, (b) EDX mapping of S, (c) EDX mapping of $\mathrm{Ni}$, (d) SSRM, (e) C-AFM, and (f) KPFM images of the composite positive electrode before charging. VCPD measured by KPFM was converted into VCPD' by adding a VOCV value of $1.16 \mathrm{~V}$. 
(a) SEM

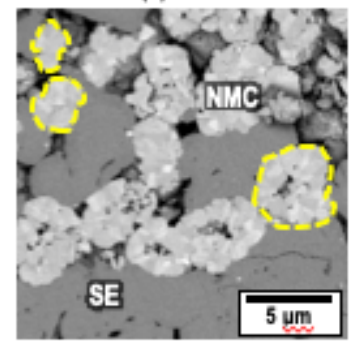

(d) SSRM

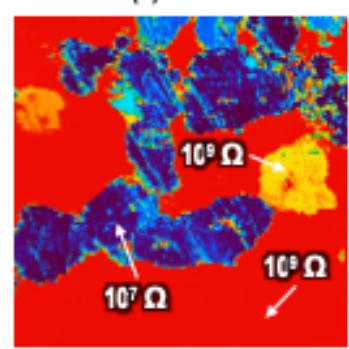

4.9E7 (b) EDX mapping

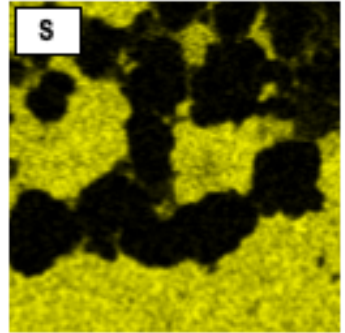

(e) C-AFM

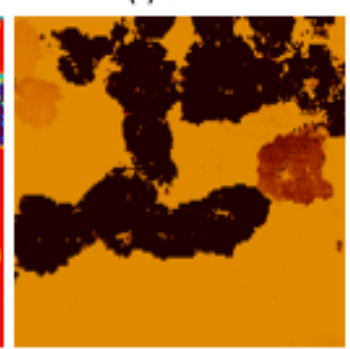

$0.5[n \mathrm{~A}]$ (c) EDX mapping

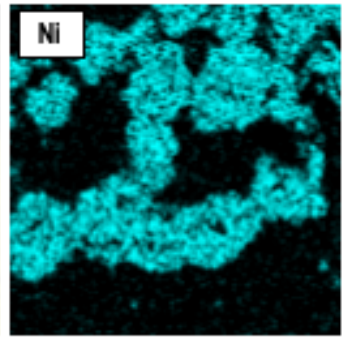

(f) KPFM

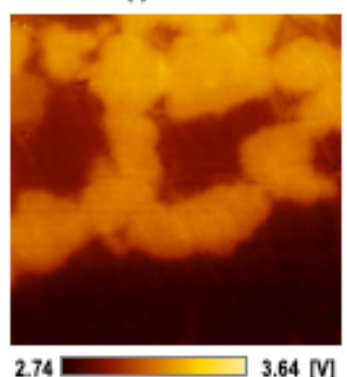

\section{Figure 3}

SPM analysis of the composite positive electrode after charging. (a) SEM, (b) EDX mapping of S, (c) EDX mapping of Ni, (d) SSRM, (e) C-AFM and (f) KPFM images of the composite positive electrode after the initial charge test. The NMC particles framed by yellow broken lines were in minimal contact with other NMC particles. VCPD measured by KPFM was converted into VCPD' by adding a VOCV value of $3.74 \mathrm{~V}$. 
(a)

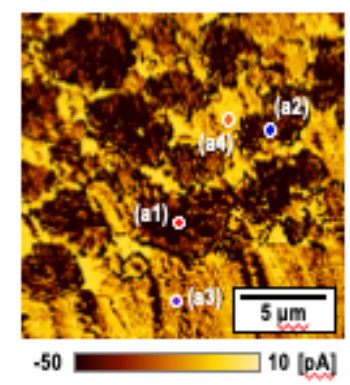

(c)

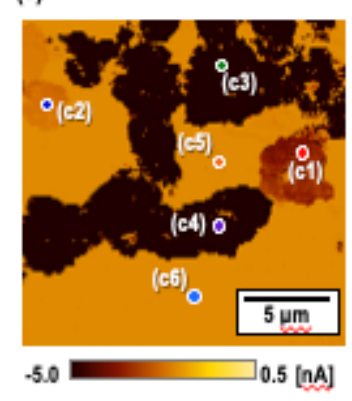

Before charge test

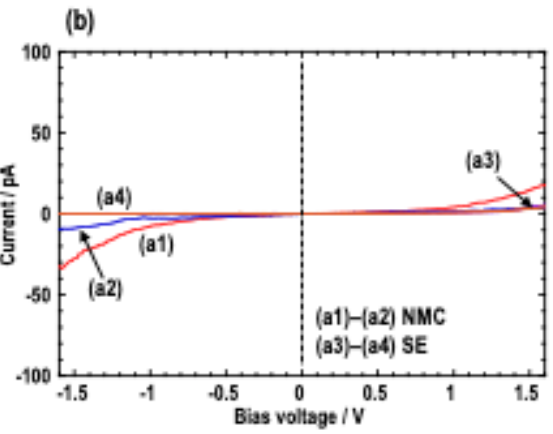

Aftercharee test

(d)

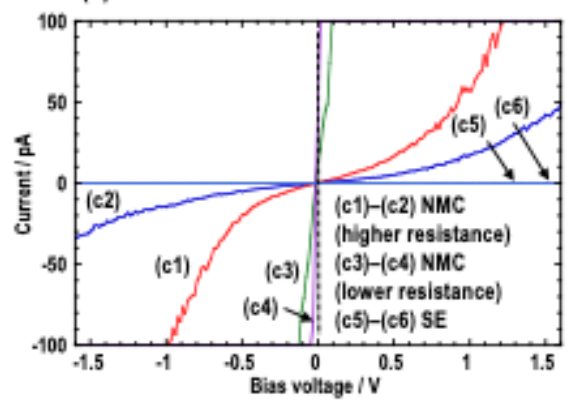

Figure 4

I-V characteristics of NMC particles and SEs in composite electrodes. (a) and (c) C-AFM images and (b) and (d) I-V curves of NMC particles and SEs in composite electrodes (a) and (b) before and (c) and (d) after charging. 

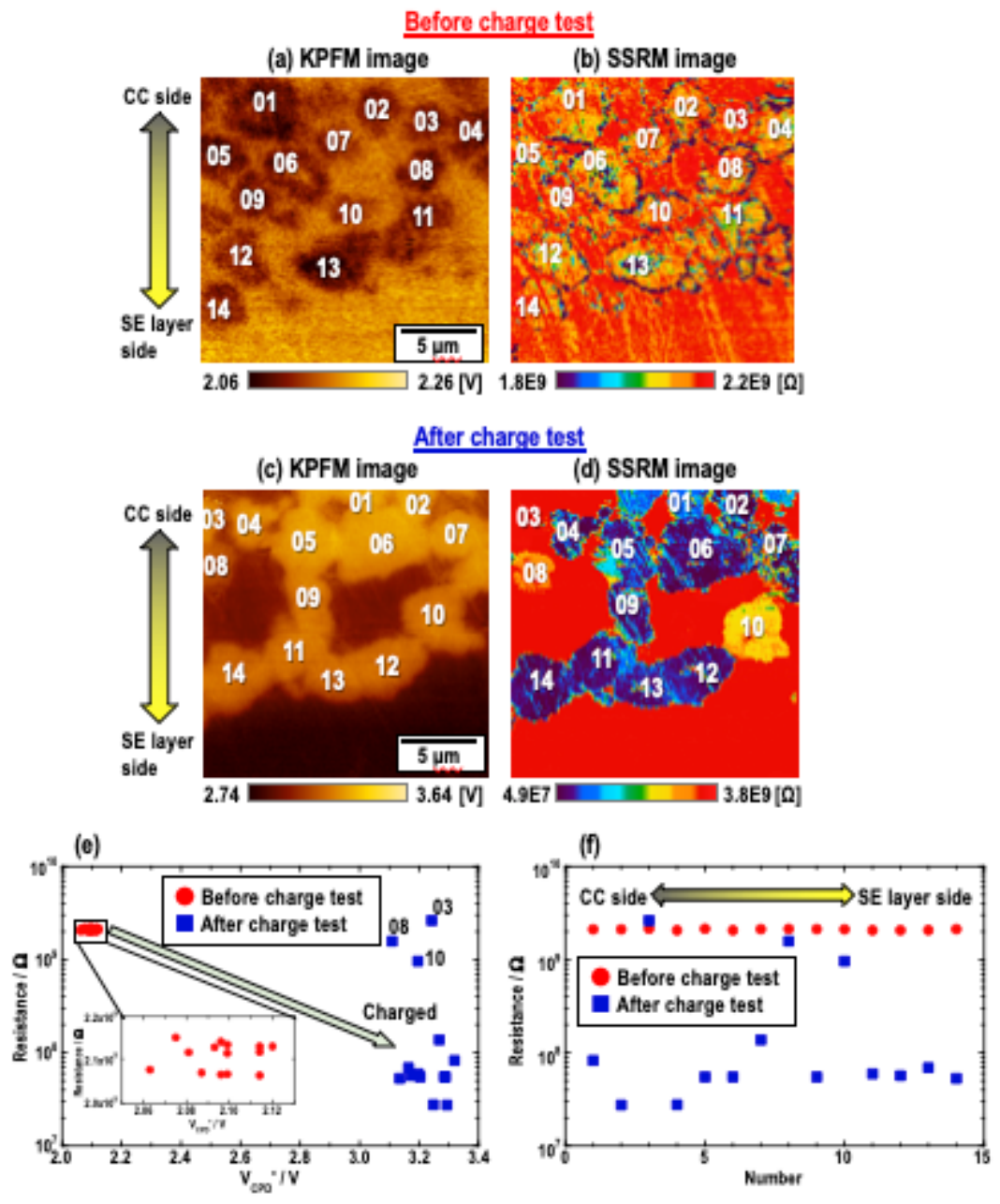

\section{Figure 5}

Potential and resistance changes of NMC particles before and after charging. (a) and (c) KPFM and (b) and (d) SSRM images of NMC particles (a) and (b) before and (c) and (d) after charging. The higher and lower numbers on the NMC particles indicate their position near the SE layer side and the CC side, respectively. (e) VCPD' and resistance of each NMC particle before ( $(\bullet)$ and after $(\boldsymbol{\square})$ charge test measured by KPFM and SSRM. The inset shows an enlarged figure of NMC particles before charging. The numbers 03, 08, and 10 correspond to the NMC particles in Figure 5(c) and (d). (f) Resistance of the NMC particles numbered in the KPFM images before and after charging.

\section{Supplementary Files}

This is a list of supplementary files associated with this preprint. Click to download.

- AFMNatureCommunotoyamaSI5.pptx

- AirPASSLBsSPMD5002020015973647511.gif 